\title{
(6) OPEN ACCESS \\ Ovarian cancer symptom awareness and anticipated time to help-seeking for symptoms among UK women
}

For numbered affiliations see end of article.

\section{Corresponding author \\ Ms Emma L Low, \\ Cancer Research UK Health \\ Behaviour Research Centre, \\ Department of Epidemiology and \\ Public Health, University College London, 1-19 Torrington Place, London WC1E 6BT, UK; \\ e.low@ucl.ac.uk}

*Present address:

Health Services Research \& Management, A224, College Building, City University London, Northampton Square, London ECIV OHB, UK

Received 29 August 2012 Revised 15 February 2013 Accepted 26 February 2013 Published Online First 24 May 2013

\section{SLinked}

- http://dx.doi.org/10.1136/ jfprhc-2013-100591

\section{ABSTRACT}

Objectives To determine levels of awareness of ovarian cancer symptoms and to identify barriers to help-seeking and predictors of a longer time to help-seeking in a UK female population-based sample.

Methods A UK population-based sample of women [ $n=1000$, including a subsample of women at higher risk due to their age $(\geq 45$ years, $n=510)$ ] completed the Ovarian Cancer Awareness Measure by telephone interview. Questions measured symptom awareness (using recall and recognition), barriers to medical helpseeking and anticipated time to help-seeking. Regression analyses identified predictors of a higher score on a scale of anticipated time to help-seeking.

Results Most women ( $58 \%$ overall sample; $54 \%$ subgroup) were unable to recall any symptoms but $99 \%$ recognised at least one. Recognition was lowest for difficulty eating and persistently feeling full. In the sample overall, higher socio-economic status and higher endorsement of practical and service barriers independently predicted a longer anticipated time to help-seeking for more symptoms. White ethnicity was an additional predictor in the older subgroup.

Conclusions This study suggests awareness of ovarian cancer symptoms is low in the UK, and varies widely between symptoms. It identifies variables that may be involved in a longer time to help-seeking for possible ovarian cancer symptoms and highlights the need for more indepth research into the factors related to time to help-seeking in real-world situations.

\section{INTRODUCTION}

Ovarian cancer is the most deadly gynaecological cancer in the $\mathrm{UK}^{1}$ responsible for more deaths than all the other gynaecological malignancies

\section{KEY MESSAGE POINTS}

- Ovarian cancer symptom awareness is low in the UK, and varies widely between symptoms.

- Socio-economic status and barrier endorsement predict longer time to helpseeking in the sample overall. Ethnicity was an additional predictor in the subgroup of women aged $\geq 45$ years.

combined. ${ }^{2}$ The 1-year survival rate for ovarian cancer in the UK is just $36 \%$, which is lower than survival rates observed in comparable countries. ${ }^{3}$ This suggests that ovarian cancer is diagnosed later in the UK than elsewhere. In fact, $60 \%$ of ovarian cancers are diagnosed at the latest stages (Stages III/IV, with associated 5-year survival rates of just 6-22\%). ${ }^{4}$ Increasing earlier stage diagnoses may therefore improve survival rates so that they are in line with or even better than those observed in other countries.

In the absence of an effective ovarian cancer screening strategy (feasibility trials are currently being undertaken, with conclusions expected in 2015), ${ }^{5}$ early-stage diagnosis is reliant upon timely patient help-seeking and appropriate action by health care professionals at patient presentation (i.e. appropriate investigations and/or referral to secondary care). However, women do not always seek help in a timely manner nor do they always receive a timely diagnosis. ${ }^{6}$ This paper focuses on the former issue.

Non-recognition of symptom seriousness (which may be the result of low symptom awareness) ${ }^{7}$ appears to be the most important patient-mediated factor 
leading to a longer time to help-seeking for ovarian cancer symptoms. ${ }^{6} 89$ The non-specific nature of ovarian cancer symptoms (such as bloating or lower back pain $)^{10}{ }^{11}$ may make it difficult to discern when a bodily change is serious, potentially contributing to non-recognition of symptom seriousness or symptom misattribution. Fear of cancer has also been found to increase time to help-seeking, while there is inconclusive evidence for the effects of age. ${ }^{8}$ Factors that may encourage prompt help-seeking include fewer perceived barriers to help-seeking ${ }^{7}$ and a personal experience of cancer. ${ }^{8}$

Much of the literature on time to help-seeking uses an arbitrary time-point cut-off to define 'delay' (such as 3 months or more). ${ }^{12}$ However, this can be problematic as a single cut-off point cannot be appropriately applied across all symptoms and cancers. ${ }^{13}$

Research to date has not specifically explored variables that might predict a longer time to help-seeking for symptoms that may indicate ovarian cancer. Doing so is key to identifying modifiable factors that could be targeted by interventions aimed at reducing time to help-seeking. To address this gap, we tested the hypotheses that lower symptom awareness, personal experience of cancer, more perceived emotional barriers (including 'fear') and/or more perceived practical barriers to help-seeking would be associated with a longer anticipated time to help-seeking for symptoms of ovarian cancer.

We were unsure of the direction of the relationship between age and help-seeking due to inconsistent or inconclusive previous findings. Consequently we made no specific hypotheses with regard to this variable. As the incidence of ovarian cancer begins to rise at around age 45 years, ${ }^{14}$ we tested the above hypotheses both in a population-based sample of women aged 16 years and over, and in a subgroup of women aged $\geq 45$ years.
METHODS

Recruitment

As part of the Target Ovarian Cancer Pathfinder Study, ${ }^{15}$ females aged $\geq 16$ years were recruited via Synovate (a global market research company) using random digit dialling. Quotas (i.e. target recruitment numbers for specific demographic groups) were used to achieve a population-based sample of women from different age groups, regions and working statuses. A total of 1000 women were interviewed over the telephone by Synovate interviewers, using landlines only. [NB. Synovate does not record the number of people approached in order to fulfill quota samples.] Respondents were interviewed for approximately 15 minutes using the Ovarian Cancer Awareness Measure (Ovarian CAM). ${ }^{16}$ The Ovarian CAM is a site-specific version of the generic Cancer Awareness Measure. ${ }^{17}$ The Ovarian CAM was specifically designed and validated to be used for both telephone and face-to-face surveys $;{ }^{16}$ we used telephone interviews as this allowed faster and relatively inexpensive data collection.

\section{Measures}

Symptom awareness

Respondents were asked one open question and a set of closed questions to measure awareness of ovarian cancer symptoms. The open question measured recall and read: "There are several warning signs and symptoms of ovarian cancer. Please name as many as you can think of". The closed questions measured recognition and read: "The following may or may not be warning signs for ovarian cancer. We are interested in your opinion". Respondents were read a list of ten known symptoms of ovarian cancer taken from the Department of Health's (DH) Key Messages for ovarian cancer for health professionals. ${ }^{10}$ Response options to each symptom were 'yes', 'no' or 'don't know'. The full list of symptoms included are given in Table 1.

Table 1 Recalled and recognised ovarian cancer symptoms

\begin{tabular}{|c|c|c|c|c|}
\hline \multirow[b]{2}{*}{ Ovarian cancer symptom } & \multicolumn{2}{|c|}{ Overall sample $(n=1000)$} & \multicolumn{2}{|c|}{ Subgroup* $(n=510)$} \\
\hline & $\begin{array}{l}\text { Recalled } \\
{[n(\%)]}\end{array}$ & $\begin{array}{l}\text { Recognised } \\
{[n(\%)]}\end{array}$ & $\begin{array}{l}\text { Recalled } \\
{[n(\%)]}\end{array}$ & $\begin{array}{l}\text { Recognised } \\
{[n(\%)]}\end{array}$ \\
\hline Persistent pain in abdomen & $238(24)$ & $837(84)$ & $132(26)$ & $409(80)$ \\
\hline Persistent pain in pelvis & $96(10)$ & $867(87)$ & $55(11)$ & $431(84)$ \\
\hline Persistent bloating & $91 \quad(9)$ & $683(68)$ & $57(11)$ & $329(65)$ \\
\hline Extreme fatigue & $35(4)$ & $703(70)$ & 17 (3) & $359(70)$ \\
\hline Increased abdominal size & $28 \quad(3)$ & $790(79)$ & 19 (4) & $393(77)$ \\
\hline Back pain & $28 \quad(3)$ & $706(71)$ & 21 (4) & $368(72)$ \\
\hline Changes in bowel habit & $24 \quad(2)$ & $569(57)$ & $16(3)$ & $280(55)$ \\
\hline Passing more urine than usual & $9(<1)$ & $450(45)$ & $4(<1)$ & $205(40)$ \\
\hline Feeling full persistently & $6(<1)$ & $450(45)$ & $5 \quad(1)$ & $253(50)$ \\
\hline Difficulty eating on most days & $8(<1)$ & $247(25)$ & $3(<1)$ & $105(21)$ \\
\hline
\end{tabular}

*Subgroup of women aged $\geq 45$ years. 
Responses to the open question were recorded verbatim and coded into a framework by Synovate interviewers. Thirty-five different codes were generated. Coding was checked for accuracy by a separate Synovate team who specialise in coding interviews. For ease of analysis, and to allow comparisons between numbers of recalled and recognised symptoms, we only report here those responses from the open question that corresponded with the closed question list of symptoms. Each open question response was dichotomised into 'recalled' (mentioned) or 'not recalled' (not mentioned). Each closed question response was dichotomised into 'recognised' (response 'yes') or 'not recognised' (responses 'no'/'don't know'). For both questions, scores were summed to give total 'symptom recall' and 'symptom recognition' scores.

\section{Barriers}

Respondents were read a list of ten barriers and the question: "Sometimes people put off going to see the doctor, even when they have a symptom that they think might be serious. These are some of the reasons people give for delaying. Could you say if any of these might put you off going to the doctor?" Responses were dichotomised into 'yes' (responses 'yes sometimes'/'yes often') and 'no' (responses 'don't know'/'no'). As previously described, ${ }^{7}$ barriers were grouped into emotional, service and practical barriers (Table 2). Responses were summed to give total scores for each group of barriers. Scores were in the range $0-4$ for emotional barriers, $0-3$ for service barriers and $0-3$ for practical barriers.

Anticipated time to help-seeking

For each symptom from the closed list, respondents were asked: 'How soon would you contact your doctor to make an appointment to discuss it?". There were ten response options on an ordinal scale ranging from '1-3 days' to 'never'. For ease of analysis we coded each time period in ascending order from 0 to 10 to measure anticipated time to help-seeking. We dichotomised the sample into those who anticipated waiting more or less time before help-seeking for each symptom. To do this, we calculated the median anticipated time to help-seeking for each symptom (using the scale from 0 to 10) and then dichotomised responses into a 'longer anticipated time to helpseeking' (>median; score $=1$ ) and a 'shorter anticipated time to help-seeking' $(\leq$ median; score $=0)$. We used a dichotomy as opposed to a continuous variable as the increments in time periods were unequal, making it an ordinal rather than an interval scale.

Dichotomising the sample in this way allowed us to identify those women who were likely to wait longer than average before seeking help for each of the symptoms, rather than focusing on the length of time to help-seeking, as we acknowledge that responses may not reflect actual responses to symptoms. Scores for each symptom were summed to create a scale of anticipated time to help-seeking (range 0-10). A higher score indicated that the respondent would anticipate waiting longer than the median before seeking help for a greater number of symptoms.

\section{Demographic characteristics}

Age was measured as a continuous variable. We created a subgroup of women aged $\geq 45$ years for analysis who were at high risk of developing ovarian cancer due to their age (incidence of ovarian cancer begins to increase at this age). ${ }^{14}$

Table 2 Endorsement of barriers to seeking medical help for a symptom that might be serious

\begin{tabular}{|c|c|c|}
\hline Barrier & $\begin{array}{l}\text { Overall }(n=1000) \\
{[n(\%)]^{*}}\end{array}$ & $\begin{array}{l}\text { Subgroupt }(n=510) \\
{[n(\%)]^{*}}\end{array}$ \\
\hline \multicolumn{3}{|l|}{ Emotional barriers } \\
\hline Worry what the GP might find & $343(34)$ & $145(29)$ \\
\hline Too scared & $209(21)$ & $77(15)$ \\
\hline Too embarrassed & $138(14)$ & $38(8)$ \\
\hline No confidence in talking to GP about symptom & $127(13)$ & $37 \quad(7)$ \\
\hline \multicolumn{3}{|l|}{ Practical barriers } \\
\hline Too busy & $296(30)$ & $102(20)$ \\
\hline Too many other things to worry about & $267(27)$ & $116(23)$ \\
\hline Difficult to arrange transport to GP & $48 \quad(5)$ & $22(4)$ \\
\hline \multicolumn{3}{|l|}{ Service barriers } \\
\hline Difficult to make an appointment with GP & $315(32)$ & $135(27)$ \\
\hline Wasting GP time & $303(30)$ & $120(24)$ \\
\hline GP difficult to talk to & $131(13)$ & $44(9)$ \\
\hline
\end{tabular}

*Respondents were able to endorse more than one barrier.

tSubgroup of women aged $\geq 45$ years.

$\mathrm{GP}$, general practitioner. 
Socio-economic status (SES) was categorised into low $($ score $=0-1)$, medium $($ score $=2)$ and high $($ score $=3)$ based on a combined index incorporating education level, car ownership and home ownership. This index was used as it is recommended for a sample including older, retired respondents. ${ }^{18}$ One point is given for owning a home, having a car and having any formal education (score range $0-3$ ).

As only $7.1 \%$ of respondents were non-White, these ethnicities were grouped together and the sample was dichotomised into 'White' or 'non-White'.

Respondents were asked whether they had known a close family member, close friend or partner with cancer. Responses were dichotomised into 'yes' $($ score $=1$ ) or 'no/don't know' (score $=0$ ) and summed to give a score of 'cancer experience' (range 0-3). Respondents were also asked whether they had experienced cancer themselves ('no' or 'yes').

\section{Analyses}

Data were analysed using SPSS version 19 (IBM Corporation, New York, NY, USA). To select variables for the multivariate models, we first used bivariate analyses (not shown here) to test for significant associations between anticipated time to help-seeking and our hypothesised predictor variables as well as demographic factors. Ethnicity, SES, practical and service barriers were associated with time to help-seeking in the sample as a whole and additionally, emotional barriers in the subgroup. Symptom knowledge and cancer experience showed no association with time to help-seeking.

Demographic variables (SES, ethnicity and age) and variables that were significantly associated with anticipated time to help-seeking were entered into two multiple linear regressions (using the sample as a whole and the subgroup of women aged $\geq 45$ years) to identify significant independent predictors of score on the scale. Linear regressions were used as the skewness and kurtosis of the outcome variable (number of symptoms participants anticipated waiting longer for) were both within normal distribution ranges $(-1$ to $1+)$. Small sampling weights were applied to adjust for non-response bias in terms of government office region, age and occupational status.

\section{RESULTS}

\section{Sample demographics}

The mean age of the sample was 47 (range 16-91) years. Most respondents were White (93\%) and around half were high SES (54\%). Almost two-thirds $(63 \%)$ of the women had known someone close with cancer, but few $(5 \%)$ had experienced cancer themselves. The subgroup of women aged $\geq 45$ years $(n=510)$ had a mean age of 62 (range 45-91) years. Again, most were White (97\%) and high SES (60\%). Nearly three quarters (72\%) had known someone with cancer.

\section{Symptom awareness}

Symptom recall was low, with $58 \%$ of the whole sample and $54 \%$ of the subgroup of women aged $\geq 45$ years unable to recall any of the 10 symptoms listed in Table 1 [mean number of symptoms recalled $(\mathrm{M})=0.6 / 10$ for the whole sample, standard deviation $(\mathrm{SD})=0.8$ and $\mathrm{M}=0.6 / 10, \mathrm{SD}=0.9$ in the older subgroup]. 'Pain in the abdomen' was the most commonly recalled symptom in both the sample overall and in the subgroup (24\% and 26\%, respectively). 'Feeling full' was the least recalled overall $(<1 \%)$ and in the subgroup it was 'difficulty eating on most days' $(<1 \%)$. Recognition scores were much higher with almost all (99\%) able to identify at least one of the 10 symptoms in the overall sample $(\mathrm{M}=6.3, \mathrm{SD}=2.2)$ and in the subgroup $(\mathrm{M}=6.1, \mathrm{SD}=2.2)$. Recognition was lowest for 'difficulty eating' $(25 \%$ in the overall sample; $21 \%$ in the subgroup) and highest for 'pain in pelvis' $(87 \%$ in the overall sample; $84 \%$ in the subgroup) (Table 1).

\section{Barriers}

Respondents endorsed few barriers to help-seeking $(\mathrm{M}=2.2 / 10, \mathrm{SD}=2.2$ for the sample overall; $\mathrm{M}=1.6 /$ $10, \mathrm{SD}=1.8$ for the subgroup). A quarter $(26 \%)$ of the overall sample anticipated no barriers, whilst the figure was higher (34\%) for women aged $\geq 45$ years. The most endorsed barrier was worry about what the general practitioner (GP) might find $(34 \%$ for the sample overall; 29\% for the subgroup) and the least endorsed was difficulty arranging transport to the GP $(5 \%$ for the sample as a whole; $4 \%$ for the subgroup) (Table 2).

\section{Anticipated time to help-seeking}

Median anticipated time to help-seeking varied by symptom. Women anticipated waiting the longest for extreme fatigue, persistent bloating, back pain, persistently feeling full and changes in bowel habit. The shortest anticipated time was for persistent abdominal pain. Patterns were the same for the overall sample and the subgroup (Figure 1 shows median time to help-seeking by symptom for the sample overall).

The regression model for the sample overall explained $6 \%$ of variance in scores on the scale of anticipated time to help-seeking [Adj $R^{2}=0.06, \quad F(5,940)=13.53$, $p<0.001]$. Endorsing more practical and service barriers and having a higher SES were significant predictors of a higher score on the scale (i.e. longer anticipated time to help-seeking for more symptoms). The standardised beta was largest for practical barriers (beta $=0.16$, $p<0.001$ ) and slightly smaller for SES (beta $=0.12$, $p<0.001$ ) and service barriers (beta $=0.10, p<0.01$ ), suggesting that perceiving more practical barriers to help-seeking has the strongest influence on anticipated time to help-seeking.

The regression model for the subgroup explained $11 \%$ of the variance in scores on the scale of anticipated time to help-seeking [Adj $R^{2}=0.11, F(6,458)$ 


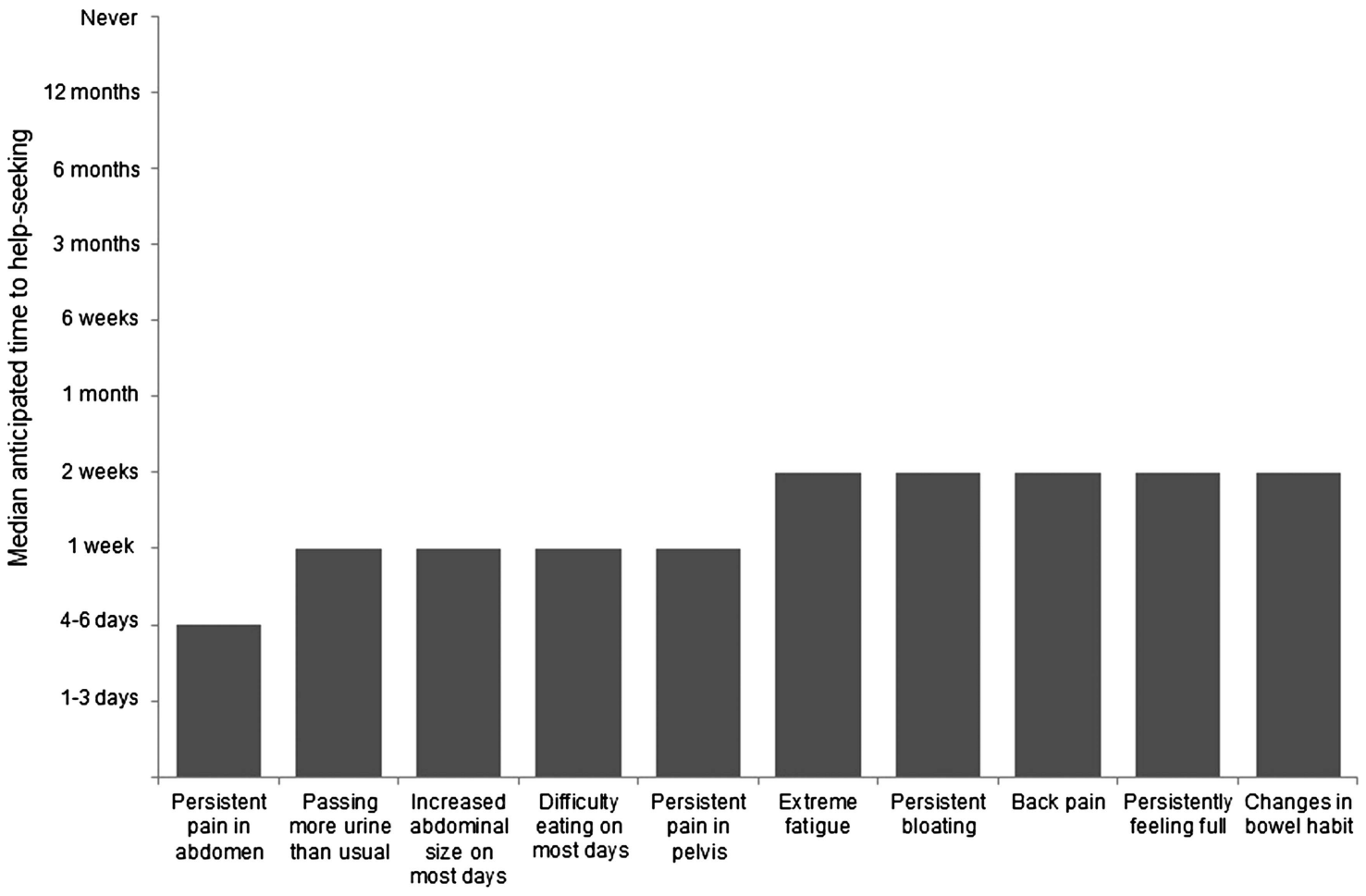

Figure 1 Median anticipated time to help-seeking by symptom for the sample overall $(n=1000)$.

$=10.56, p<0.001]$. Endorsing more practical and service barriers, having a higher SES and being of a White ethnicity all significantly predicted a higher score. The standardised beta was largest for SES (beta $=0.22, p<0.001$ ) and smaller for service barriers (beta $=0.17, p=0.001$ ), practical barriers (beta $=0.13$, $p<0.01$ ) and ethnicity (beta $=0.12, p<0.01$ ), suggesting that, in the subgroup of women aged $\geq 45$ years, SES has the strongest influence on anticipated time to help-seeking. Table 3 contains both regressions.

Table 3 Multiple linear regression analyses showing predictors of longer time to help-seeking

\begin{tabular}{|c|c|c|c|c|c|c|}
\hline \multirow[b]{2}{*}{ Predictor } & \multicolumn{3}{|c|}{$\begin{array}{l}\text { Overall sample } \\
(n=946)^{*}\end{array}$} & \multicolumn{3}{|c|}{$\begin{array}{l}\text { Subgroup } \\
(n=504)^{*}\end{array}$} \\
\hline & Betat & SE & $p$ & Betat & SE & $p$ \\
\hline Constant & - & - & 0.014 & - & - & 0.529 \\
\hline Age & 0.06 & 0.03 & 0.064 & 0.01 & 0.05 & 0.802 \\
\hline Ethnicity & 0.07 & 0.04 & 0.075 & 0.12 & 0.04 & 0.008 \\
\hline SES & 0.12 & 0.05 & 0.000 & 0.22 & 0.05 & 0.000 \\
\hline Practical barriers & 0.16 & 0.04 & 0.000 & 0.13 & 0.05 & 0.008 \\
\hline Service barriers & 0.10 & 0.05 & 0.005 & 0.17 & 0.05 & 0.001 \\
\hline Emotional barriers & - & - & - & 0.04 & 0.05 & 0.413 \\
\hline
\end{tabular}

Overall sample $\operatorname{Adj} R^{2}=0.06, p<0.001$; subgroup $\operatorname{Adj} R^{2}=0.11, p<0.001$. NB. Ethnicity is a categorical variable, coded as Non-white $=0$, White $=1$ and SES is an ordinal variable, coded as Low=0, Mid=1 and High=2.

* Sample sizes are smaller due to missing data. †Standardised.

SE, standard error; SES, socio-economic status.

\section{DISCUSSION}

To our knowledge, this study is the first to systematically measure anticipated time to help-seeking for ovarian cancer symptoms and to identify variables associated with a longer time to help-seeking specifically for this cancer in a UK sample. This work is important, as 1-year survival rates for ovarian cancer are considerably lower in the UK than in comparable countries, ${ }^{3}$ suggesting that this cancer is diagnosed later in the former and it has been argued that early diagnosis may be improved through prompt patient presentation with symptoms. ${ }^{19}$

This is also the first study that has attempted to measure awareness of a comprehensive list of ovarian cancer symptoms in a UK population and to identify symptoms that are more versus less well known. Given that previous research has found that misattributing symptoms of ovarian cancer to benign or coexisting conditions led to a longer time to help-seeking, ${ }^{6}$ it is important that we are aware of the current levels of awareness and can identify areas for improvement in knowledge.

Ovarian cancer symptom recall was very low (overall $\mathrm{M}=0.6 / 10$; subgroup $\mathrm{M}=0.6 / 10$ ), whereas recognition was considerably higher, reflecting previous findings using recall and recognition to measure cancer symptom awareness. ${ }^{72}$-22 This disparity may be a product of the increased complexity of the cognitive processes required for recall, compared to those involved in recognition, which may, on occasion, be influenced by guessing. ${ }^{23}$ Nevertheless, on average women were still only able to recognise just over half 
of the symptoms (overall $\mathrm{M}=6.3 / 10$; subgroup $\mathrm{M}=6.1 / 10$ ) when prompted, suggesting that there is a need for improvement in awareness of symptoms potentially indicative of ovarian cancer.

Furthermore, as symptoms for ovarian cancer can be non-specific ${ }^{10} 11$ it might be pertinent for any interventions designed to increase awareness to focus on the characteristics of the symptoms experienced as well as their presence. For example, there is evidence that symptoms such as abdominal pain, pelvic pain, bloating, constipation, and increased abdominal size are significantly more severe and frequent in women with ovarian cancer than in women in a general clinic population. $^{24}$

Symptoms for which the lowest recognition was observed were frequent urination (overall $=45 \%$; subgroup $=40 \%$ ), feeling full (overall $=45 \%$; subgroup $=40 \%$ ) and difficulty eating (overall $=25 \%$; subgroup $=21 \%)$. Research suggests that these symptoms (as well as abdominal and pelvic pain) are present in earlier-stage ovarian cancers; ${ }^{25-28}$ consequently consideration of these symptoms as potential ovarian cancer symptoms could aid earlier diagnoses. That awareness was lowest for these symptoms, then, is concerning. On average a GP will only see one case of ovarian cancer every 5 years, ${ }^{29} 30$ which may reduce the likelihood of GP consideration of ovarian cancer in a patient presenting with such symptoms. However, if awareness were higher in women overall, this could increase the chances of the patient herself considering ovarian cancer, and therefore being able to highlight this to her GP upon presentation.

In contrast to our hypothesis, we found no association between symptom awareness and anticipated time to help-seeking. This finding does not necessarily mean that symptom awareness has no influence on time to help-seeking, however. Symptom awareness may influence attribution or appraisal behaviour (which has been shown to be related to time to help-seeking), ${ }^{8}$ rather than having a direct relationship with time to helpseeking. This relationship should be explored further to aid our understanding of the complexities of helpseeking behaviour for ovarian cancer symptoms.

It was promising that women anticipated seeking help quickest for persistent abdominal pain as this is one of the three most important symptoms highlighted to health professionals in the DH's Key Messages on ovarian cancer, ${ }^{10}$ and has been found in up to $49 \%$ of early-stage (I/II) invasive epithelial ovarian cancer cases ${ }^{31}$ (90\% of ovarian cancers are this type). ${ }^{32}$ Previous research exploring symptom presentation in primary care found that abdominal pain was one of the most common symptoms reported. ${ }^{24}$ This finding, taken together with our own results, suggests that women may feel comfortable presenting with abdominal pain and consequently may not anticipate a reason to wait before seeking help for this symptom.
Women in both the sample overall and the subgroup at increased risk (those aged $\geq 45$ years) anticipated waiting the longest for extreme fatigue, persistent bloating, back pain, persistently feeling full and changes in bowel habit. All of these symptoms are mentioned in the DH's Key Messages, ${ }^{10}$ which highlight persistent bloating and persistently feeling full as two of the three most important symptoms. Abdominal bloating and loss of appetite (represented here as persistently feeling full) have been found to have positive predictive values (PPVs) for ovarian cancer of $0.3 \%$ and $0.6 \%$, respectively. When paired, these two symptoms have a PPV of $3.3 \%$, second only to the pairing of loss of appetite and abdominal distension (PPV $>5 \%) .{ }^{26}$ Although these PPVs are not high (possibly owing to a high frequency of these symptoms in the general population), ${ }^{33} 34$ bloating or feeling full have been found to be present in over $44 \%$ of early stage disease. ${ }^{31}$

Further, Olson and colleagues ${ }^{25}$ found that $71 \%$ of ovarian cancer patients reported experiencing bloating and/or fullness in the 6-12 months prior to diagnosis; similar results to those reported by Goff et al. 3 years later. ${ }^{24}$ Consequently, if a patient presents with both symptoms, it may help a GP to more accurately either diagnose or refer, particularly if the patient is aged $\geq 45$ years, given that these symptoms are more likely to be associated with ovarian cancer with increasing age. ${ }^{14}$ Encouraging earlier help-seeking for these two symptoms specifically, particularly in older women, may impact on diagnoses of less advanced disease.

The amount of variance explained by the regression models was relatively small, suggesting that there are other factors that are important in anticipated time to help-seeking. However we did identify some important predictor variables. In the overall sample and in the subgroup, more perceived practical barriers predicted a higher score on the scale of anticipated time to helpseeking (indicating a longer time to help-seeking for more symptoms), supporting previous research exploring help-seeking for 'generic' cancer symptoms. ${ }^{35}$ In contrast to the findings in the latter research, we also found that endorsing more perceived service barriers was a significant predictor of a higher score on the scale of anticipated time to help-seeking, whereas endorsing more emotional barriers was not. This finding may be due to the low awareness that these symptoms could be indicative of ovarian cancer, leading women to assume that these symptoms were not significant enough to merit the difficulty of arranging a doctor's appointment.

An interesting finding was that women from lower SES and non-White ethnic groups anticipated seeking help earlier for more symptoms of ovarian cancer than those from higher SES and White ethnic backgrounds. This finding reflects earlier research ${ }^{76}$ and there is evidence that these relationships between ethnicity and SES and help-seeking also apply to actual help-seeking behaviour. ${ }^{37}$ However, women from lower SES and 
non-White ethnic backgrounds are more frequently diagnosed with later-stage cancer compared to those from higher SES and White backgrounds. ${ }^{38-40}$ The disparity in primary care help-seeking and stage of diagnosis between White and non-White ethnicities may be a result of GP time to referral and diagnosis, ${ }^{37}$ which in turn may be a product of the lower ovarian cancer incidence rates observed in Asian and Black ethnicities when compared to the White ethnic group. ${ }^{41}$ Ovarian cancer cases are rare in primary care $;{ }^{29}{ }^{30}$ if few of those cases are in women of non-White ethnicities, this may lead to a much reduced possibility that a GP will even consider an ovarian cancer diagnosis in these women even if they present promptly with symptoms. In contrast, however, no such difference has been observed in incidence for ovarian cancer between SES groups. ${ }^{42}$ Consequently, the disparity in SES groups warrants further investigation.

\section{Study strengths and limitations}

This study was strengthened by the use of a validated tool to measure awareness and anticipated helpseeking behaviours. If this tool is utilised in future research, it will allow researchers to identify shifts or differences in patterns of help-seeking by accurately comparing these data with other time periods or other countries. Although the sample was not population representative, we collected data from a range of women from different age and demographic groups. Consequently, it is likely that the relationships found in our results would apply to the UK population.

A potential limitation of this study is the measurement of help-seeking intention for a hypothetical symptom as opposed to actual help-seeking behaviour for an existing symptom. Although it is unlikely that anticipated help-seeking would map exactly onto actual behaviour, this was a pragmatic method that allowed us to explore the correlates of anticipated help-seeking in a large sample. Measuring true helpseeking behaviour is notoriously difficult, whether retrospective or prospective designs are used.

A second limitation is that questions were asked within the context of ovarian cancer and it is likely that women anticipated faster help-seeking than would occur in a real-life situation where the symptom might not be appraised as a warning sign of cancer. For example, the longest median anticipated time to help-seeking was 2 weeks. However, we limited the impact of this issue by looking at median time to help-seeking for each symptom and thus explored the relative speed of help-seeking behaviour for each symptom, rather than the time period itself. Further, DH guidelines for patients ${ }^{29}$ do not give advice on how long women should wait before seeking medical help for symptoms potentially indicative of ovarian cancer. Although $\mathrm{DH}^{10}$ and National Institute for Health and Clinical Excellence ${ }^{43}$ guidelines mention persistency as a key feature for health professionals to look for, for a number of symptoms (such as pelvic or abdominal pain, abdominal distension and difficulty eating or feeling full quickly) they do not define 'persistency', therefore making it difficult to say how long women should wait before seeking help for these symptoms.

A further limitation applicable to our measure was that we did not investigate frequency, severity or novelty of hypothetical symptoms, and their impact on intention to seek help. Doing so would have been interesting, as symptoms that are frequent, severe and novel are more likely to indicate ovarian cancer. ${ }^{8} 10242743$ Finally, coding of the open recall items was carried out by Synovate researchers, and as some symptoms could potentially be viewed as being similar (for example 'persistent bloating' and 'increased abdominal size') it is possible that there was some coding error. This may have resulted in our estimates of knowledge being too conservative.

Quota sampling was used because it is an efficient and practical method of collecting data. However, this method limits the generalisability of the findings. Despite this, the study was well-powered and novel and it provides us with an indication of the factors that may influence time to help-seeking, as well as an idea of the relative levels of awareness of different ovarian cancer symptoms in a UK female population.

\section{CONCLUSIONS}

This study suggests that there is low awareness of ovarian cancer symptoms in this population and variation in anticipated help-seeking behaviour for these symptoms. Women anticipated waiting longest for persistent bloating and persistently feeling full, symptoms that are both associated with ovarian cancer, and have been found in just under half of early-stage cases.

Although barriers may be important in predicting help-seeking, there may be more important variables involved that were not measured here. Consequently, there is a need for more in-depth, exploratory research to identify these factors.

\section{Author affiliations}

${ }^{1} \mathrm{PhD}$ Student, Cancer Research UK Health Behaviour Research Centre, Research Department of Epidemiology and Public Health, University College

London, London, UK

${ }^{2}$ Senior Research Associate, Cancer Research UK

Health Behaviour Research Centre, Research

Department of Epidemiology and Public Health, University College London, London, UK

${ }^{3}$ Professor of Gynaecological Cancer (UCL Institute of Women's Health) and Consultant Gynaecologist (UCLH NHS Trust), Gynaecological Cancer Research Centre, Institute of Women's Health, University College London, London, UK

${ }^{4}$ Chief Executive, Target Ovarian Cancer, London, UK 
${ }^{5}$ Director of Public Affairs and Communications, Target Ovarian Cancer, London, UK

${ }^{6}$ Senior Research Associate, Cancer Research UK Health Behaviour Research Centre, Research Department of Epidemiology and Public Health, University College London, London, UK

Funding This research was funded by Target Ovarian Cancer.

\section{Competing interests None declared.}

Open Access This is an Open Access article distributed in accordance with the Creative Commons Attribution Non Commercial (CC BY-NC 3.0) license, which permits others to distribute, remix, adapt, build upon this work non-commercially, and license their derivative works on different terms, provided the original work is properly cited and the use is non-commercial. See: http://creativecommons.org/licenses/by-nc/3.0/

\section{REFERENCES}

1 Office for National Statistics. Cancer Incidence and Mortality in the United Kingdom, 2008-10. 2012. http://www.ons.gov.uk/ ons/dcp171778_289890.pdf [accessed 12 December 2012].

2 Cancer Research UK. Ovarian Cancer Mortality Statistics. 2013. http://www.cancerresearchuk.org/cancer-info/cancerstats/types/ ovary/mortality/\#Gynaecological [accessed 18 January 2013].

3 Coleman M, Forman D, Bryant $\mathrm{H}$, et al. Cancer survival in Australia, Canada, Denmark, Norway, Sweden, and the UK, 1995-2007 (the International Cancer Benchmarking

Partnership): an analysis of population-based cancer registry data. Lancet 2011;377:127-138.

4 Cancer Research UK. Ovarian Cancer Survival Statistics. 2011. http://info.cancerresearchuk.org/cancerstats/types/ovary/ survival/ [accessed 20 December 2011].

5 Menon U, Gentry-Maharaj A, Hallett R, et al. Sensitivity and specificity of multimodal and ultrasound screening for ovarian cancer, and stage distribution of detected cancers: results of the prevalence screen of the UK Collaborative Trial of Ovarian Cancer Screening (UKCTOCS). Lancet Oncol 2009;10:327-340.

6 Evans J, Ziebland S, McPherson A. Minimizing delays in ovarian cancer diagnosis: an expansion of Andersen's model of "total patient delay". Fam Pract 2007;24:48-55.

7 Robb K, Stubbings S, Ramirez A, et al. Public awareness of cancer in Britain: a population-based survey of adults. Br J Cancer 2009;101(S2):S18-S23.

8 Macleod U, Mitchell ED, Burgess C, et al. Risk factors for delayed presentation and referral of symptomatic cancer: evidence for common cancers. Br J Cancer 2009;101(S2): S92-S101.

9 Smith LK, Pope C, Botha JL. Patients' help-seeking experiences and delay in cancer presentation: a qualitative synthesis. Lancet 2005;366:825-831.

10 Department of Health. Key Messages for Ovarian Cancer for Health Professionals. Department of Health Publications. Policy and Guidance. 2009. http://www.dh.gov.uk/en/ Publicationsandstatistics/Publications/PublicationsPolicy AndGuidance/DH_110534 [accessed 20 December 2011].

11 National Institute for Health and Clinical Excellence (NICE). Referral Guidelines for Suspected Cancer. 2005. http://www. nice.org.uk/nicemedia/pdf/cg027niceguideline.pdf [accessed 13 January 2013].
12 Facione NC. Delay versus help seeking for breast cancer symptoms: a critical review of the literature on patient and provider delay. Soc Sci Med 1993;36:1521-1534.

13 Walter F, Webster A, Scott S, Emery J. The Andersen Model of Total Patient Delay: a systematic review of its application in cancer diagnosis. J Health Serv Res Policy 2011;17:110-118.

14 Cancer Research UK. Ovarian Cancer Risk Factors. 2011. http://info.cancerresearchuk.org/cancerstats/types/ovary/ riskfactors/\#Age [accessed 20 December 2011].

15 Target Ovarian Cancer. Target Ovarian Cancer Pathfinder Study First Results Report. Target Ovarian Cancer. 2009. http://www.targetovariancancer.org.uk/core/core_picker/ download.asp? $\mathrm{id}=335$ [accessed 3 September 2011].

16 Simon A, Wardle J, Grimmett C, et al. Ovarian and cervical cancer awareness: development of two validated measurement tools. J Fam Plann Reprod Health Care 2012;38:164-174

17 Stubbings S, Robb K, Waller J, et al. Development of a measurement tool to assess public awareness of cancer. $\mathrm{Br} \mathrm{J}$ Cancer 2009;101(S2):S13-S17.

18 Wardle J, Farrell M, Hillsdon M, et al. Smoking, drinking, physical activity screening uptake health inequalities. In: Gordon D, Shaw M, Dorling D, Davey-Smith G (eds), Inequalities in Health. Bristol, UK: Policy Press 1999:213-239.

19 Richards MA. The National Awareness Early Diagnosis Initiative in England: assembling the evidence. $\mathrm{Br} \mathrm{J} \mathrm{Cancer}$ 2009;101:S1-S4.

20 Waller J, Robb K, Stubbings S, et al. Awareness of cancer symptoms and anticipated help seeking among ethnic minority groups in England. Br J Cancer 2009;101(S2):S24-S30.

21 Simon AE, Juszczyk D, Smyth N, et al. Knowledge of lung cancer symptoms and risk factors in the UK: development of a measure and results from a population-based survey. Thorax 2012;67:426-432.

22 Low EL, Simon AE, Lyons J, et al. What do British women know about cervical cancer symptoms and risk factors? Eur J Cancer 2012;48:3001-3008.

23 Waller J, McCaffery K, Wardle J. Measuring cancer knowledge: comparing prompted and unprompted recall. $\mathrm{Br} \mathrm{J}$ Psychol 2004;95:219-234.

24 Goff B, Mandel L, Melancon C, et al. Frequency of symptoms of ovarian cancer in women presenting to primary care clinics. JAMA 2004;291:2705-2712.

25 Olson S, Mignone L, Nakraseive C, et al. Symptoms of ovarian cancer. Obstet Gynecol 2001;98:212-217.

26 Hamilton W, Peters TJ, Bankhead C, et al. Risk of ovarian cancer in women with symptoms in primary care: population based case-control study. BMJ 2009;339:b2998.

27 Goff BA, Mandel LS, Drescher CW, et al. Development of an ovarian cancer symptom index. Cancer 2007;109:221-227.

28 Lataifeh I, Marsden D, Robertson G, et al. Presenting symptoms of epithelial ovarian cancer. Aust N Z J Obstet Gynaecol 2005;45:211-214.

29 NHS Choices. Know the Symptoms of Ovarian Cancer - Live Well. 2012. http://www.nhs.uk/Livewell/preventing-cancer/Pages/ ovarian-cancer-symptoms.aspx [accessed 25 January 2013].

30 Office for National Statistics. Cancer 1971-1997. London; 1999.

31 Rossing MA, Wicklund KG, Cushing-Haugen KL, et al. Predictive value of symptoms for early detection of ovarian cancer. J Natl Cancer Inst 2010;102:222-229.

32 Cancer Research UK. Types of Ovarian Cancer. 2012. http:// cancerhelp.cancerresearchuk.org/type/ovarian-cancer/about/ types-of-ovarian-cancer [accessed 7 June 2012]. 
33 Pitts MK, Heywood W, Ryall R, et al. High prevalence of symptoms associated with ovarian cancer among Australian women. Aust N Z J Obstet Gynaecol 2011;51:71-78.

34 Agréus L, Svürdsudd K, Nyrén O, et al. The epidemiology of abdominal symptoms: prevalence and demographic characteristics in a Swedish adult population: a report from the Abdominal Symptom Study. Scand J Gastroenterol 1994;29:102-109.

35 Simon AE, Waller J, Robb K, et al. Patient delay in presentation of possible cancer symptoms: the contribution of knowledge and attitudes in a population sample from the United Kingdom. Cancer Epidemiol Biomarkers Prev 2010;19:2272-2277.

36 Adamson J, Ben-Shlomo Y, Chaturvedi N, et al. Ethnicity, socioeconomic position gender - do they affect reported health-care seeking behaviour? Soc Sci Med 2003;57:895-904.

37 Morris S, Sutton M, Gravelle H. Inequity, inequality in the use of health care in England: an empirical investigation. Soc Sci Med 2005;60:1251-1266.
38 Downing A, Prakash K, Gilthorpe MS, et al. Socioeconomic background in relation to stage at diagnosis, treatment, survival in women with breast cancer. Br J Cancer 2007;96:836-840.

39 Morris CR, Sands MT, Smith LH. Ovarian cancer: predictors of early-stage diagnosis. Cancer Causes Control 2010;21: 1203-1211.

40 Schwartz KL, Crossley-May H, Vigneau FD, et al. Socioeconomic status stage at diagnosis for five common malignancies. Cancer Causes Control 2003;14:761-766.

41 National Cancer Intelligence Network, Cancer Research UK. Cancer Incidence and Survival by Major Ethnic Group, England, 2002-2006. 2009.

42 National Cancer Intelligence Network. Cancer Incidence by Deprivation England, 1995-2004. 2009.

43 National Institute for Health and Clinical Excellence (NICE). Ovarian Cancer: The Recognition and Initial Management of Ovarian Cancer. 2011. http://guidance.nice.org.uk/CG122/ Guidance/pdf/English [accessed 11 February 2013]. 